An ocean undercurrent, a thermocline, a free surface, with waves: a problem in classical fluid mechanics

R. S. Johnson

To cite this article: R. S. Johnson (2015) An ocean undercurrent, a thermocline, a free surface, with waves: a problem in classical fluid mechanics, Journal of Nonlinear Mathematical Physics 22:4, 475-493, DOI:

https://doi.org/10.1080/14029251.2015.1113042

To link to this article: https://doi.org/10.1080/14029251.2015.1113042

Published online: 04 January 2021 


\title{
An ocean undercurrent, a thermocline, a free surface, with waves: a problem in classical fluid mechanics
}

\author{
R. S. Johnson \\ School of Mathematics \& Statistics, Newcastle University \\ Newcastle upon Tyne, NE1 7RU, UK \\ robin.johnson@newcastle.ac.uk
}

Received 30 June 2015

Accepted 1 October 2015

\begin{abstract}
We describe a problem that can be tackled more-or-less routinely using the ideas of classical fluid mechanics, but it is a complex flow and even the linearised problem involves considerable algebraic complexity. The presentation here emphasises the approach that we adopt in order to formulate an accessible model of such a flow. The physical background is carefully described: the Equatorial Undercurrent, a particular phenomenon of the Pacific Ocean, the thermocline and the waves on both the free surface and on the thermocline. One of the results of this careful approach, coupled with a solution of the linearised problem for arbitrary wave numbers, is that we are able to provide well-grounded explanations for many of the fundamental processes that are relevant, and observed, in this region of the oceans; the result is a successful application of elementary principles. In the context of this system, we can describe the various types of wave dynamics, depending on wavelength, and also the differences between eastward and westward propagation. It is gratifying that the results of such a simple theory correspond closely to the observations reported in the literature. Of more interest, perhaps, from the theoretical-fluids viewpoint, is that the development leads directly to the prediction of critical layers and to a procedure for their analysis, which we outline here (and critical layers and their associated flows have been observed in the Pacific Ocean). Further, other possible rôles of nonlinearity are immediately accessible, such as wave evolution, for which we provide only an introduction, but this is sufficient to hint at tantalising prospects for further work.
\end{abstract}

Keywords: Ocean undercurrent; nonlinearity; waves; asymptotic expansions.

2000 Mathematics Subject Classification: 35R35, 76B15, 76B55

\section{Introduction}

The theoretical studies that revolve around fluid mechanics have excited mathematicians, physicists and engineers for over 200 years. Every conceivable (and probably many that we cannot yet imagine) motion of a fluid - air, rivers, oceans, in pipes, in arteries, and so much more - is described by our governing equations. The real surprise is that the Euler equation alone is so successful: for example, with incompressibility, we have most of the possible motions of water, with compressibility we have supersonic flow. The reasons for this, by and large, are because what we see (usually something very turbulent) is, in an average sense, what Euler predicts (even if the rigorous development of this is lacking), and viscosity, usually, produces effects over very large time and distance scales. (Of course, there are important exceptions, but many flows can be characterised as above.) One of the challenges for the modern researcher is to see how far these classical ideas and techniques can be taken. Indeed, we often find that considerable insight is available from a fairly routine application of a standard approach to a new - probably quite complicated - fluid system. The motion 
of the oceans is a case in point; only over the last few decades have we begun to collect sufficient data to allow an attempt to analyse and understand some of what is observed.

Thus we are beginning to appreciate the factors that contribute to the climate, climate change and specific phenomena (such as El Niño). Nevertheless, there is no realistic possibility of ever being able to analyse flows, on different scales, and embodying e.g. wind-driven surface waves, underwater currents, upwelling and downwelling, thermal, density and salinity stratification, and so on, ocean wide. However, the study of certain, rather limited classes of flows is possible, and these might lead to new and important conclusions about the details of the various processes that are involved. The aim of this article is to show how familiar ideas can be used to study a quite complicated flow in ocean dynamics. The emphasis here will be on the modelling and approximation techniques that are to be employed. So we will set-up and define the problem with some care, and leave a lot of the technical - and quite messy - details to one side. (A comprehensive discussion of this problem, with gratifyingly good agreement with the available data, can be found elsewhere; we will provide some references at the appropriate stage.) Our first task, therefore, is to describe the nature (and physics) of the problem that we shall examine; we will give a few key references to the background ideas in oceanography, for the interested reader.

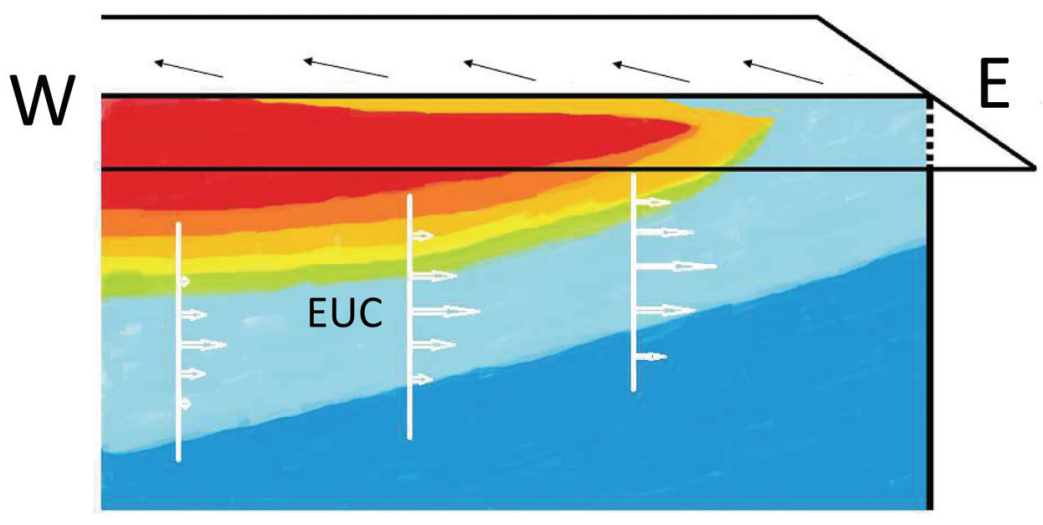

Fig. 1. A sketch of the ocean along the Pacific Equator; the warmer water above the thermocline is coloured yellow-red, and this extends upwards and to the East. On the surface, the flow is away from the equator and to the West; this is indicated on the northern side of the Equator only, for simplicity. The EUC velocity profile at three stations is included in the sketch.

We must start with a brief description of the nature of some rather particular flows that are encountered in one of our oceans. It is observed that, in a band of about $2^{\circ}$ latitude around the Pacific Equator, the ocean possesses some quite unique features: a significant fluid stratification, resulting in a pycnocline/thermocline, and a depth-dependent current which goes from a westward flow near the surface to a quite significant flow to the East, situated a little below the surface. Then, superimposed on this structure, there is a wide variety of wave-propagation modes. And so to the details: near the surface, there is a layer of relatively warm water separated from a deeper layer of denser, cold water by the thermocline; the densities above and below can reasonably be taken 
as constants. Further, also in a near-surface layer, and within about $150 \mathrm{~km}$ on each side of the Equator, there is the westward current (driven by the prevailing trade winds); in this same region of the Equator, but confined to depths of no more than about $100 \mathrm{~m}$, lies the Equatorial Undercurrent (EUC); this is an eastward-flowing jet whose core resides on the thermocline. Then deeper down, at depths in excess of about $240 \mathrm{~m}$, we have, essentially, an abyssal layer of still water; a sketch of this flow configuration is shown in Figure 1. (Some general background, providing a description of these flows, can be found in [17] and [12].) All this constitutes a prescribed, pre-existing state, when viewed as a problem in fluid mechanics. We then have, superimposed on this given flow field, a wave system. There are gravity waves on the surface, and corresponding oscillations of the thermocline; further, there are oscillations with an amplitude of ten or twenty metres at the thermocline (which is perhaps at a mean depth of $120 \mathrm{~m}$ ) that produce no measurable disturbance at the surface. There are waves of many different wave lengths. A successful theoretical study of this problem will produce predictions for the speeds of the waves (as a function of wave length), and for the coupling between the waves on the surface and on the thermocline. (A description of some of the wave modes, and corresponding data, can be found in [8], which also gives a good survey of the methods often employed in oceanography.)

The approach normally adopted in oceanographic circles starts from a set of model equations, typically those that are generated by reduced-gravity, shallow-water, linear theory; this is usually coupled with the assumption that there is an infinitely deep, motionless layer below the thermocline. It is clear that this will have a number of drawbacks. Some of the physical elements (which may be of importance) are removed altogether, and there is no possibility of using nonlinearity in any way e.g. for examining critical layers or for looking at the nonlinear evolution of the waves. In part, these criticism can be overcome if any exact solutions of some related, nonlinear system can be constructed; this has been done in this case (see e.g. $[5,6,10]$ but these solutions have a restricted applicability e.g. not valid for all wave numbers, or not valid everywhere). Numerical solutions are not our concern but, in any event, these are not well-adapted to the determination, and description, of the various processes that are present, and they are necessarily restricted, in each computation, to a particular wave length. Clearly, an analytical solution valid for arbitrary wave numbers, and containing a range of the relevant physical parameters, is far preferable. Further, if this solution is based on a linearisation - and it will be - then recourse to the original, underlying, nonlinear equations will enable some discussion of the effects of nonlinearity in a very direct and precise manner.

It is evident from our description of the physical system that it is quite involved and, even though there is much retained in the model, some simplifications are necessary. Most importantly, we assume that the flow is purely two-dimensional: we suppress any motion, and any variation, away from the Equator. (We will explain the 2D model in more detail below, but we can report that it is possible to find three-dimensional flows which are exact solutions of a slightly reduced, but fully nonlinear, system; this work is ongoing.) From our introductory comments, it is no surprise that our fundamental assumption is that we can model the fluid by Euler's equation, so that we are dealing with an inviscid fluid (although we do include vorticity in the background state, as we shall describe). That the Reynolds number is extremely large for these types of geophysical flows is well known (see [16]), and so this assumption is altogether reasonable. We shall carefully describe the model, the non-dimensionalisation and the scaling, leading to the prescription of the linear system. This is not the place to give an extensive and detailed description of the solution to the linear problem; however, a very broad outline of the main results will be presented (and 
further information can be found in [7]). We will, however, include a section describing how the full, nonlinear set of equations can be used to investigate the nature of the critical layers that appear here (which appears as an appendix in [7]), and begin the exploration of a new area for this problem: how the nonlinear evolution of the waves can be examined.

\section{The model and governing equations}

The development of our model is based on the Euler equation and the equation of mass conservation for constant density. This latter equation is still appropriate for our problem, which involves density stratification, because we will consider a jump in conditions across the thermocline/pycnocline: we assume constant density in each of the two regions. We use a coordinate frame that rotates with the Earth, which has its origin on the Equator with the $\bar{x}$-axis horizontally due East, the $\bar{y}$ axis horizontally due North and the $\bar{z}$-axis vertically upwards. (We will use the over bar to denote physical variables; the notation will be simplified shortly, when we non-dimensionalise.) Thus our coordinate system is defined in the tangent plane on the Equator; see Figure 2.

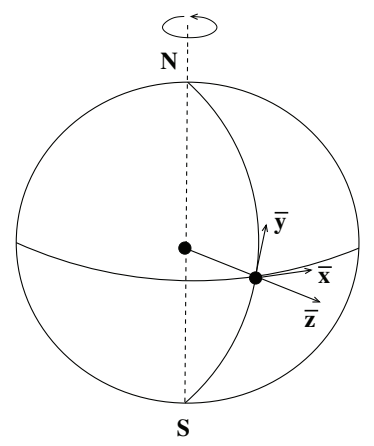

Fig. 2. The rotating frame of reference, a tangent plane fixed to a point on the Equator.

The two governing equations may therefore be written (in the usual notation) as

$$
\frac{\mathrm{D} \overline{\mathbf{u}}}{\mathrm{D} \bar{t}}+2 \overline{\boldsymbol{\Omega}} \times \overline{\mathbf{u}}=-\frac{1}{\bar{\rho}} \bar{\nabla} \bar{p}+\overline{\mathbf{F}} \quad \text { and } \quad \bar{\nabla} \cdot \overline{\mathbf{u}}=0
$$

where the Coriolis term is based on the rotational angular speed of the Earth $\left(|\overline{\mathbf{\Omega}}|=\bar{\Omega} \approx 7.29 \times 10^{-5}\right.$ $\left.\operatorname{rad~s}^{-1}\right), \overline{\mathbf{F}}$ is the body force (simply generated by gravity here) and the density $(\bar{\rho})$ takes one of two constant values (below/above $\approx 1.005$; this and other useful information can be found in [14]). However, in order to make the problem manageable, we need to simplify the contribution from the Coriolis effect; this is accomplished by invoking the standard (oceanographic) approximation close to the Equator. So, for small values of $\bar{y} / R$ (where $R$ is an average radius of the Earth), we have

$$
2 \overline{\boldsymbol{\Omega}} \times \overline{\mathbf{u}} \sim 2 \bar{\Omega}(\bar{w}-\bar{y} \bar{v} / R, \bar{y} \bar{u} / R,-\bar{u}) \quad \text { where } \quad \overline{\mathbf{u}}=(\bar{u}, \bar{v}, \bar{w}) .
$$

The retention of the terms in $\bar{y} / R$ here constitutes the ' $\beta$-plane' approximation and necessarily leads to a 3D structure for the flow field; we will restrict the discussion here to $2 \mathrm{D}$, and so these terms are ignored (the factor $\bar{y} / R$ being only about 0.024 even $150 \mathrm{~km}$ away from the Equator). This further simplification is the ' $f$-plane' approximation, and is regarded as an adequate model within about $2^{\circ}$ of the Equator; see [15] for some general background, and [20] for an extensive 
discussion of the mathematical merits of these approximations; the consequences of relaxing these approximations are considered in [9]. Because we wish to discuss purely 2D flows, we require the additional assumption that there is no flow in the meridional direction i.e. $\bar{v}=0$. Finally, we will be considering some perturbation of a background state; this flow is given by $(\bar{U}(\bar{z}), 0)$ for some suitable $\bar{U}$, which is maintained by the pressure $\bar{P}(\bar{z})$. We therefore replace $\bar{u}$ by $\bar{U}+\bar{u}$ and $\bar{p}$ by $\bar{P}+\bar{p}$, and so produce our set of governing equations:

$$
\begin{aligned}
& \bar{u}_{\bar{t}}+(\bar{U}+\bar{u}) \bar{u}_{\bar{x}}+\bar{w}\left(\bar{U}^{\prime}+\bar{u}_{\bar{z}}\right)+2 \bar{\Omega} \bar{w}=-\frac{1}{\bar{\rho}} \bar{p}_{\bar{x}}, \\
& \bar{w}_{\bar{t}}+(\bar{U}+\bar{u}) \bar{w}_{\bar{x}}+\bar{w} \bar{w}_{\bar{z}}-2 \bar{\Omega}(\bar{U}+\bar{u})=-\frac{1}{\bar{\rho}}\left(\bar{P}^{\prime}+\bar{p}_{\bar{z}}\right)-\bar{g}, \\
& \bar{u}_{\bar{x}}+\bar{w}_{\bar{z}}=0,
\end{aligned}
$$

all written in the usual notation. The constant density of the fluid, $\bar{\rho}$, is replaced by $(1+r) \bar{\rho}$ for the fluid below the thermocline, where $r$ is the small positive constant alluded to above.

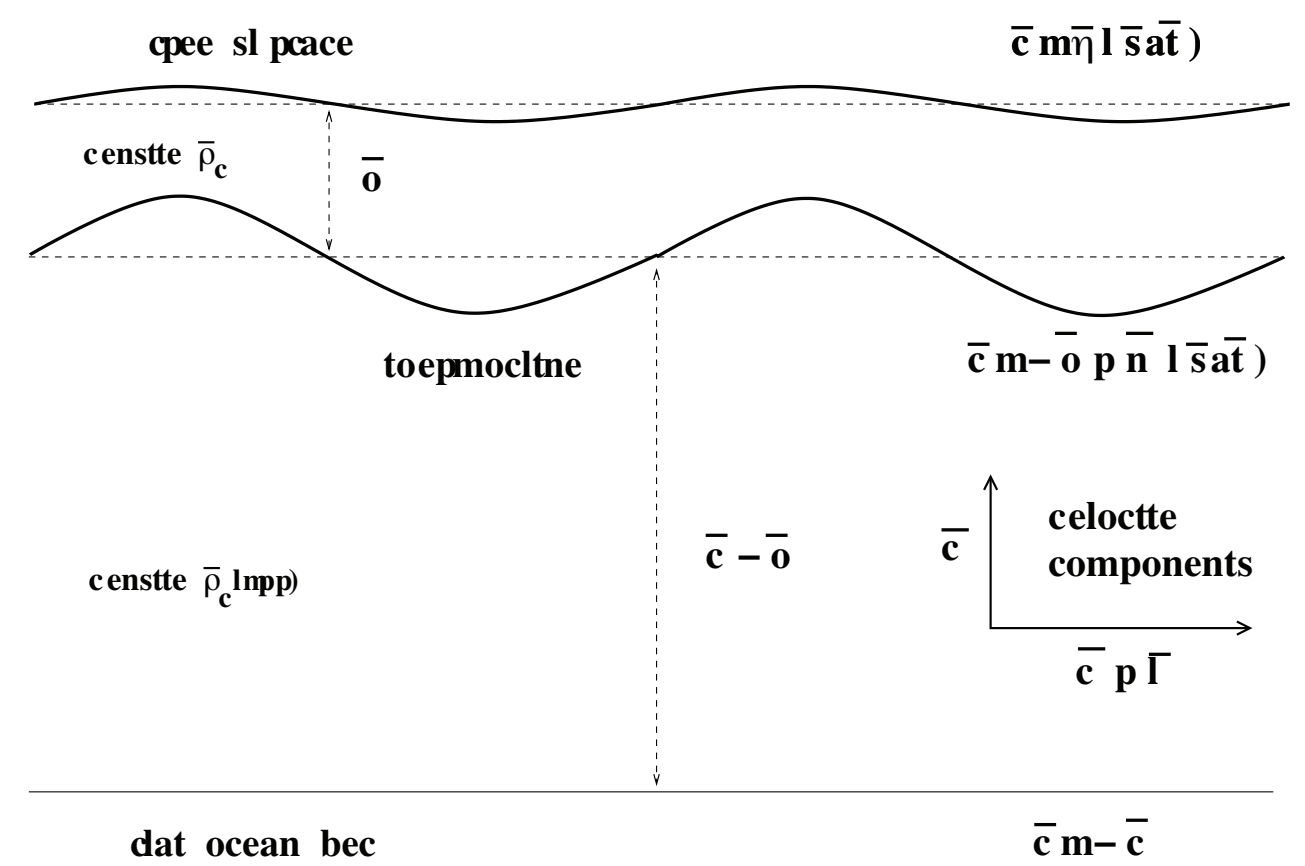

Fig. 3. A sketch of the cross-section of the fluid domain at a fixed latitude; the upper surface is a free surface, the thermocline separates regions of different, constant density, and the lower boundary is a flat, impermeable, rigid bed. Waves propagate, at the same speeds, on the free surface and on the thermocline.

We now state the appropriate boundary conditions for this problem; at the free surface $(\bar{z}=$ $\bar{\eta}(\bar{x}, \bar{t})$ ), we have a pressure (dynamic) condition and a kinematic condition (so that particles of the surface remain in the surface: there is no mixing):

$$
\bar{P}+\bar{p}=\bar{P}_{a} \quad \text { on } \quad \bar{z}=\bar{\eta}(\bar{x}, \bar{t}),
$$

and

$$
\bar{w}=\bar{\eta}_{\bar{t}}+(\bar{U}+\bar{u}) \bar{\eta}_{\bar{x}} \quad \text { on } \quad \bar{z}=\bar{\eta}(\bar{x}, \bar{t})
$$


where $\bar{P}_{a}$ is the constant pressure of the atmosphere above the ocean. At the thermocline $(\bar{z}=$ $-\bar{h}+\bar{H}(\bar{x}, \bar{t})$ ), with the constant $\bar{h}$ used to denote the undisturbed level of the thermocline, we have the kinematic condition (so there is no mixing here, either)

$$
\bar{w}=\bar{H}_{\bar{t}}+(\bar{U}+\bar{u}) \bar{H}_{\bar{x}} \quad \text { on } \quad \bar{z}=-\bar{h}+\bar{H}(\bar{x}, \bar{t}) ;
$$

we must also impose the continuity of pressure across the thermocline (to avoid infinite accelerations of the thermocline). Finally, there is to be no flow through the flat, horizontal, impermeable bed $($ at $\bar{z}=-\bar{d})$

$$
\bar{w}=0 \quad \text { on } \quad \bar{z}=-\bar{d} .
$$

This general configuration is shown in Figure 3.

\section{Non-dimensionalisation, scaling and linearisation}

In order to proceed, and to be clear about the various approximations that we invoke, we nondimensionalise the system described by equations (2.1)-(2.7). To accomplish this, we require suitable general scales that are appropriate to this problem. Here, we elect to use as the fundamental length scale, $\bar{h}$, the mean depth of the undisturbed thermocline; we do not introduce a separate scale for horizontal and vertical motions - as is the usual practice in many discussions of the classical water-wave problem - because we will consider both short and long waves. Associated with this is the natural speed scale $\sqrt{\bar{g}} \bar{h}$ (which happens to be the speed of long gravity waves over a depth $\bar{h}$, but this does not restrict our discussion in any way). The non-dimensionalisation of the pressure is defined in terms of the pressure difference over the depth $\bar{h}$, and uses the density above the thermocline. Finally, we need some measure of the amplitudes of the waves, whether on the surface or on the thermocline; let a typical or average amplitude (of which ever happens to be the larger) be $\bar{a}$.

These scales are now used to define a set of non-dimensional variables:

$$
\begin{cases}\bar{z}=\bar{h} z, \quad \bar{x}=\bar{h} x, \quad \bar{t}=\bar{h} t / \sqrt{\bar{g} \bar{h}}, \quad \bar{\eta}=\bar{a} \eta, \quad \bar{H}=\bar{a} H, \\ (\bar{U}+\bar{u}, \bar{w})=\sqrt{\bar{g} \bar{h}}(U+u, w), \quad \bar{P}+\bar{p}=\overline{\rho_{0}} \bar{g} \bar{h}(P+p) .\end{cases}
$$

Thus the absence of the over-bar indicates that the variables are now the non-dimensional counterparts of those with the over bar. The set of equations (2.1)-(2.7) therefore becomes

$$
\begin{aligned}
& u_{t}+[U(z)+u] u_{x}+w\left[U^{\prime}(z)+u_{z}\right]+2 \Omega w=-p_{x}, \\
& w_{t}+[U(z)+u] w_{x}+w w_{z}-2 \Omega[U(z)+u]=-\left[P^{\prime}(z)+p_{z}\right]-1, \\
& u_{x}+w_{z}=0,
\end{aligned}
$$

with

$$
\begin{array}{lll}
P(z)+p=\overline{P_{a}} /(\bar{\rho} \bar{g} \bar{h})=P_{a} & \text { on } & z=\varepsilon \eta, \\
w=\varepsilon\left\{\eta_{t}+[U(z)+u] \eta_{x}\right\} & \text { on } & z=\varepsilon \eta,
\end{array}
$$

and

$$
\begin{aligned}
& w=\varepsilon\left\{H_{t}+[U(z)+u] H_{x}\right\} \quad \text { on } \quad z=-1+\varepsilon H, \\
& w=0 \quad \text { on } \quad z=-d,
\end{aligned}
$$


where $\varepsilon=\bar{a} / \bar{h}$, which is our fundamental small parameter. This measures, in an average sense, the size of the waves that we shall discuss. The other parameters introduced here are $\Omega=\bar{\Omega} \bar{h} / \sqrt{\bar{g}} \bar{h}$, which now describes the Earth's rotation in this model, and $d=\bar{d} / \bar{h}$, the total (non-dimensional) depth. It follows directly from the boundary conditions that $p$ and $w$, and hence $u$, are proportional to $\varepsilon$. Thus we replace the set $(u, w, p)$ by $\varepsilon(u, w, p)$ : we have introduced scaled variables, which represent the perturbation of the background state, the size of the perturbation being measured by $\varepsilon$. Finally, the pressure that supports the background flow, $U(z)$, is defined by the solution to

$$
-2 \Omega U(z)=-P^{\prime}(z)-1
$$

with

$$
P=P_{a} \quad \text { on } \quad z=0
$$

above the thermocline, and

$$
-2 \Omega U(z)=-\frac{1}{1+r} P^{\prime}(z)-1
$$

below. The solution for $P(z)$ then enables the continuity of pressure across the thermocline to be applied, and the condition on $p$ determined; this is included in the set of equations below. With this behaviour incorporated in the equations (3.2)-(3.8), the full prescription of the problem, written in non-dimensional, scaled variables, becomes

$$
\begin{aligned}
& u_{t}+[U(z)+\varepsilon u] u_{x}+w\left[U^{\prime}(z)+\varepsilon u_{z}\right]+2 \Omega w=-p_{x}, \\
& w_{t}+[U(z)+\varepsilon u] w_{x}+\varepsilon w w_{z}-2 \Omega u=-p_{z}-1, \\
& u_{x}+w_{z}=0
\end{aligned}
$$

with

$$
\begin{aligned}
& P(z)+\varepsilon p=P_{a} \quad \text { on } \quad z=\varepsilon \eta, \\
& w=\eta_{t}+[U(z)+\varepsilon u] \eta_{x} \text { on } \quad z=\varepsilon \eta,
\end{aligned}
$$

and

$$
\begin{aligned}
& w=H_{t}+[U(z)+\varepsilon u] H_{x} \quad \text { on } \quad z=-1+\varepsilon H, \\
& p_{a}-p_{b}=-r(1-2 \Omega U(z)) H \quad \text { on } \quad z=-1+\varepsilon H,
\end{aligned}
$$

with

$$
w=0 \quad \text { on } \quad z=-d,
$$

where the subscripts 'a' and 'b' denote 'above' and 'below', respectively.

We now invoke the familiar procedure of applying Taylor expansions about $z=0$, and about $z=$ -1 , in the boundary conditions, thereby simplifying these considerable for the purposes of defining, in particular, the linear problem. (Such mappings to a rectangular region, which is equivalent to employing Taylor expansions, can be rigorously justified. Here, we comment that this generates a valid first approximation to the problem in which the nonlinearity remains weak; the higherorder terms in $\varepsilon$ then contribute uniformly small corrections to the linear solution. We will give an introduction to the rôle of nonlinearity in the evolution of the waves, over suitable long scales, later.) 
Putting all this together, and taking $\varepsilon \rightarrow 0$, leads to the statement of the linearised problem:

$$
\begin{aligned}
& u_{t}+U(z) u_{x}+w U^{\prime}(z)+2 \Omega w=-p_{x}, \\
& w_{t}+U(z) w_{x}-2 \Omega u=-p_{z}, \\
& u_{x}+w_{z}=0
\end{aligned}
$$

with

$$
\begin{aligned}
& p=(1-2 \Omega U(z)) \eta \quad \text { on } \quad z=0, \\
& w=\eta_{t}+U(z) \eta_{x} \quad \text { on } \quad z=0,
\end{aligned}
$$

and

$$
\begin{aligned}
& w=H_{t}+U(z) H_{x} \quad \text { on } \quad z=-1, \\
& p_{a}-p_{b}=-r(1-2 \Omega U(z)) H \quad \text { on } \quad z=-1,
\end{aligned}
$$

with

$$
w=0 \quad \text { on } \quad z=-d .
$$

At the thermocline, we have a jump in density, so we must note that equations (3.20) and (3.21), when written for the region below the thermocline, require $p$ to be replaced by $p /(1+r)$.

\section{The model}

The description of the problem is completed by the specification of the pure-current background flow. Our aim is to show that we can develop a model, based on elementary principles, that describes this flow. In this case, it must include an equatorial undercurrent (to the East) and a surface current (usually regarded as wind-driven) to the West. A simple choice which accomplishes this is

$$
U(z)=\left\{\begin{array}{l}
-V-z(V+W) /(1-l) \text { for }-1+l<z \leq 0 \\
W \text { for }-1-m<z \leq-1+l \\
W(z+n) /(n-1-m) \text { for }-n<z \leq-1-m, \\
0 \text { for }-d \leq z \leq-n,
\end{array}\right.
$$

where we require $0<l<1, n>1+m, m>0$, and $d>n$; the undisturbed thermocline is placed at $z=-1$. This profile describes an equatorial undercurrent with its maximum (a uniform $W>0$ to the East) in $-1+l \geq z \geq-1-m$; the speed then reduces to zero (occurring at $z=-n$ ), and below this the fluid is stationary. At the surface there is a maximum speed of $V$ ( $>0$ to the West); an example of this flow is incorporated within Figure 4. This model contains 6 adjustable parameters, so not only can many variants of this type of shape be examined, but also we will be able to identify the most significant factors that contribute to the properties of the waves. (For information, typical values of the six parameters, as they relate to the Pacific Equatorial flow, are

$$
V=0.014, \quad W=0.029, \quad m=l=0.33, \quad n=2, \quad d=33
$$

this is based on data provided in [12].)

We see that the profile has introduced a suitable vorticity distribution, and this is fixed within this model (because we are using the Euler equation); thus any perturbation, e.g. superimposed waves, 
must necessarily be associated with an irrotational flow, so we must add to equations (3.20)-(3.27) the condition

$$
u_{z}-w_{x}=0
$$

throughout the fluid.

\section{Solution of the linear problem}

The structure of this linear problem makes clear that the solution of (3.20)-(4.2) involves the examination of five regions (I-V): $0 \geq z>-1+l,-1+l>z>-1,-1>z>-1-m,-1-m>z>-n$, $-n>z \geq-d$, which are represented in Figure 4. It turns out to be natural, and convenient, to start from the bottom (Region V) and work upwards. We seek a single, general harmonic mode with wave number $k$ (which we take to be positive throughout) then, because the system is linear, we may later add together any number of such modes. One possibility is to add an infinity of modes or, equivalently, to integrate over the wave number in order to construct a more general solution (and this would allow the inclusion of some suitable initial data). Our philosophy, however, is to assume that suitable initial data exist that give rise to the solution that we seek here (in fact, the familiar approach in this type of fluids problem). In this particular context, it might be helpful to think of using just two modes; typically, these could be a short-wavelength gravity wave (at the surface) combined with a very-long-wavelength wave (which is likely to be the driver for the wave on the thermocline).

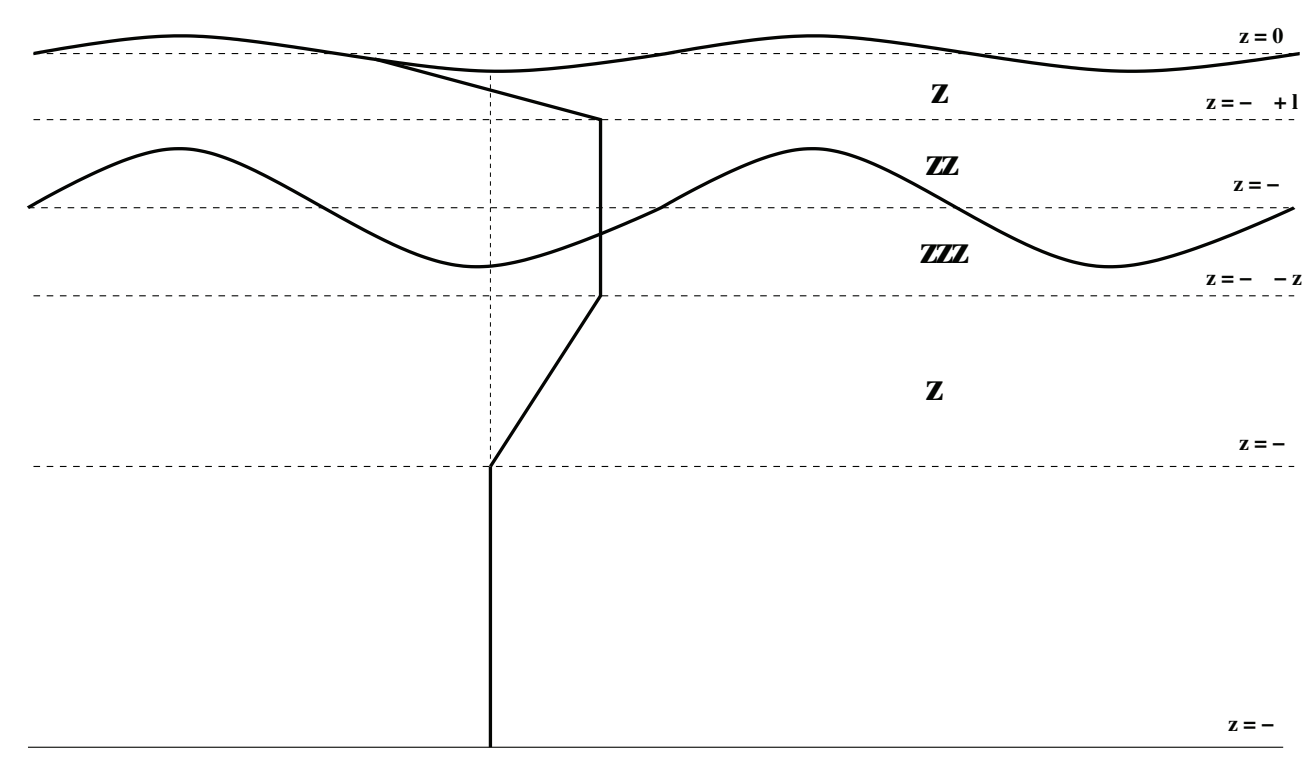

Fig. 4. A sketch of the five regions required in the description of the solution, together with a typical velocity profile of the background flow.

The analysis of this problem, although quite routine, is very lengthy and, in places, quite cumbersome. This is not the place to describe the intricacies; for the interested reader, all the background discussion, and much of the fine detail of the calculation, can be found in [7]. The emphasis that we want to put on this approach is that it follows an altogether familiar route, even if the details are 
a little more taxing that is usually encountered. One reason for the complexity is that the analysis is valid for arbitrary wave lengths; this is coupled with the inclusion of various parameters, which opens the possibility for investigations of many slightly different scenarios (and also to the use of extensive numerical work, for those so minded).

One fairly simple way forward is to look at various limits of the wave length. It turns out that there are three cases of interest: short waves (but these are not relevant to any significant motion of the thermocline), intermediate long waves (which are long with respect to our length scale - the depth of the thermocline - but short as compared with the depth of the ocean), and long waves (which are long relative to the depth of the ocean). These observations alone give an indication that the final expressions are rich with useful information. Rather than itemise all the results that can be accessed, we give a flavour of what can be done in two cases that are of the most relevance in this context: the two variants of long waves. This will give us the opportunity to indicate what is involved in producing some useful results.

\subsection{Intermediate long waves}

These waves are fairly long, but not too long; we define them by the limiting processes: $k \rightarrow 0$ and $k d \rightarrow \infty$. This describes waves that are long with respect to our scale length (the average depth of the thermocline), but short relative to the depth of the ocean (measured by $d$, in non-dimensional variables). The resulting expression for the wave speeds in this limit is still quite daunting. However, we take advantage of the fact that a number of the parameters that we have introduced take small numerical values, namely $r, \Omega, V$ and $W$. Even this is not altogether routine: we find that the crucial parameter in this problem is $r / V^{2}$, which turns out to be quite large for the Pacific EUC. Using this as the basis for an approximation procedure, we find that the wave speeds are accurately represented by

$$
c \sim \pm \sqrt{r}+W-\frac{1}{2}(1-l)(V+W), \quad c \sim W-l(V+W)+\left(\frac{1-l}{r}\right)(V+W)^{3} .
$$

These speeds correspond to physical speeds of about $2.7 \mathrm{~ms}^{-1}$ (eastwards), $1.7 \mathrm{~ms}^{-1}$ (westwards) and $0.55 \mathrm{~ms}^{-1}$ (eastwards), respectively. Reported values (see [8]) for these speeds are $2.5 \mathrm{~ms}^{-1}$ to the East and $0.53 \mathrm{~ms}^{-1}$ to the West (but this latter result is for very long waves, which we consider below). The other mode of propagation to the East is at a speed $\left(0.55 \mathrm{~ms}^{-1}\right)$ which corresponds to the appearance of a critical level below the surface (and critical levels have been observed in equatorial flows with wavelengths of a few hundred metres; see [19]).

An important prediction from these theories is for the amplitude ratio of the two sets of waves (surface:thermocline $=a: \Delta$ ); for small $r$ (this all we need to use here) we obtain, for these long waves,

$$
a \sim-r \Delta
$$

which is the same for all three wave modes. The attenuation at the surface is therefore significant: large-amplitude waves on the thermocline will not be seen at the surface. Further, the opposite sign in (5.2) demonstrates that the coupled internal and surface waves are out of phase, their crests and troughs interchanging. 


\subsection{Long waves}

In this wave-length regime, defined by $k \rightarrow 0$ with $k d \rightarrow 0$, we produce an even more complicated dispersion relation than previously (although still somewhat simpler than the original, general dispersion relation for arbitrary $k$ !). The underlying problem turns out to be a quintic in the wave speed, but - as earlier - we can quite readily obtain approximations to these roots (and simply taking $r$ and $V$ to be small, and $d$ large, is sufficient). Thus we find

$$
c \sim W \pm \sqrt{\frac{l m r}{l+m}(1-2 \Omega W)}, \quad c \sim-V+\frac{(1-l) V^{2}}{d(V+W)}, \quad c \sim \pm \sqrt{d} .
$$

where the last three results are solutions of the corresponding Burns condition which, in this context, takes the form

$$
(1-2 \Omega c) \int_{-d}^{0} \frac{\mathrm{d} z}{(U(z)-c)^{2}}=1
$$

see, for example, [11].

The first two speeds in (5.3) are, in physical variables, approximately $1.9 \mathrm{~ms}^{-1}$ and $0.11 \mathrm{~ms}^{-1}$; these correspond well with the reported data: $1.7 \mathrm{~ms}^{-1}$ (see [2,13]) and $0.12 \mathrm{~ms}^{-1}$ (see [3]). Indeed, this second speed is a critical speed, and [3] provides evidence of thermoclinic eddies associated with this mode for waves in the western equatorial Pacific Ocean. The attenuation of this pair of wave solutions can be represented, for small $r$, by

$$
\frac{a}{\Delta} \sim-\frac{r m^{2}}{(l+m)(1+m)} ;
$$

one immediate observation is that this depends, in quite a detailed manner, on the structure of the underlying flow. This factor, however, is always small for the configuration of the EUC, and the surface and thermoclinic oscillations are again out of phase.

The third speed given in (5.3) produces an approximate (physical) speed of $0.51 \mathrm{~ms}^{-1}$ to the West, and the reported speed for very long waves (see [8]) is about $0.5 \mathrm{~ms}^{-1}$; we note that critical levels might appear here. The associated amplitude ratio for these waves is

$$
\frac{a}{\Delta} \sim \frac{V^{2}}{V^{2}+d}
$$

when suitably approximated for the parameter values that we have used for the EUC. These waves also exhibit a very significant attenuation at the surface, so disturbances on the thermocline will not be observed at the surface. These waves, however, in contrast to all the other modes of propagation that we have found, are in phase. (The last pair of speeds in (5.3) describe very fast gravity waves (with speeds of about $200 \mathrm{~ms}^{-1}$ ), which would be appropriate for large-scale gravity waves, such as tsunamis, propagating across the ocean - see the discussion in [4]; these are of no interest in the context of oscillations of the thermocline.)

Of course, we can give only the smallest indication of what can be extracted from this linear theory, but all the indicators are that the results for various extremes of wavelength produce results that are in good agreement with the observations. We can expect that there will be a corresponding success for other wavelengths, although to obtain the relevant information may require a numerical investigation of the general dispersion relation (which is available in [7]). 


\section{The rôle of nonlinearity}

One of the primary aims of this work is to show that a careful formulation of the problem - even though the main intention may be, initially, to develop and examine a linear theory - enables the direct inclusion of nonlinear terms and effects, when they are appropriate. This procedure is not based on any ad hoc re-statement of the governing equations (perhaps by simply adding a few terms that seem reasonable); rather, it is a matter of constructing a different, but related, asymptotic solution of the original, scaled equations. Here, we indicate how these ideas can be applied by looking at two problems: the structure of critical layers and the nonlinear evolution of moderately long waves (although for this latter calculation we will limit how much we reproduce, but we do highlight various issues).

\subsection{Critical layers}

We have mentioned (in Section 5) that some of the wave speeds that we have found correspond to the appearance of critical levels below the surface; indeed, the eddies associated with such flows have been observed in the Pacific equatorial regions. We will outline how this problem can be described using our full set of governing equations, (3.12)-(3.19) with (4.2). (The only option, within our model, for providing a structure in the neighbourhood of the critical levels is to use the nonlinearity of the Euler equation. If viscosity had been included, then we could consider developing a viscous structure around the critical level; for a discussion of some of these issues see $[1,16]$.) Let us suppose that the speed of one of the wave components, $c$, is such that $-V<c<W$, then a critical level exists in Region I. Indeed, if $0<c<W$, then there is a second, corresponding critical level in Region IV. We will analyse the situation for the critical level in Region I; a critical level in Region IV can be described in an analogous fashion.

In Region I, our equations can be written

$$
\begin{aligned}
& u_{t}+(-V-\beta z+\varepsilon u) u_{x}+w\left(-\beta+\varepsilon u_{z}\right)+2 \Omega w=-p_{x} \\
& w_{t}+(-V-\beta z+\varepsilon u) w_{x}+\varepsilon w w_{z}-2 \Omega u=-p_{z} \\
& u_{x}+w_{z}=0
\end{aligned}
$$

where $\beta=(V+W) /(1-l)$; we seek a solution based on the propagation of a steady wave, with the associated characteristic $\xi=x-c t$, which gives

$$
\begin{aligned}
& (-c-V-\beta z+\varepsilon u) u_{\xi}+w\left(-\beta+\varepsilon u_{z}\right)+2 \Omega w=-p_{\xi} \\
& (-c-V-\beta z+\varepsilon u) w_{\xi}+\varepsilon w w_{z}-2 \Omega u=-p_{z} \\
& u_{\xi}+w_{z}=0
\end{aligned}
$$

Now the solution valid away from the critical layer, based on the linear problem with one harmonic component (wave number $k$ ), can be expressed in terms of the stream function as

$$
\psi_{z} \sim-V-\beta z+\varepsilon\left(A \mathrm{e}^{k z}-B \mathrm{e}^{-k z}\right) \cos (k \xi),
$$

and so

$$
\psi \sim-V z-\frac{1}{2} \beta z^{2}+\varepsilon\left(A \mathrm{e}^{k z}+B \mathrm{e}^{-k z}\right) \frac{1}{k} \cos (k \xi) .
$$


We define $z_{c}$, the critical level, by $c+V+\beta z_{c}=0$, and write $z=z_{c}+\delta Z$ (for small $\delta$ ) to give

$$
\psi \sim-V z_{c}-\frac{1}{2} \beta z_{c}^{2}-\delta\left(V+\beta z_{c}\right) Z-\delta^{2} \frac{1}{2} \beta Z^{2}+\varepsilon\left(A \mathrm{e}^{k z_{c}}+B \mathrm{e}^{-k z_{c}}+\mathrm{O}(\delta)\right) \frac{1}{k} \cos (k \xi) .
$$

We now introduce the stream function, $\Psi$, in the moving frame i.e. associated with $u-c$; thus we obtain

$$
\Psi+\text { const. } \sim-\frac{1}{2} \delta^{2} \beta Z^{2}+\varepsilon\left(A \mathrm{e}^{k z_{c}}+B \mathrm{e}^{-k z_{c}}\right) \frac{1}{k} \cos (k \xi),
$$

an asymptotic representation which breaks down in the classical way for critical layers: the appropriate choice is $\delta=\mathrm{O}(\sqrt{\varepsilon})$, describing the size of the neighbourhood of $z=z_{c}$ where the critical layer has structure.

This leads to a rescaling of the equations, based on the variables relevant to the solution valid in this layer, so we write

$$
z=z_{c}+\sqrt{\varepsilon} Z \text { and } u=\frac{1}{\sqrt{\varepsilon}} \hat{U}(Z, \xi ; \varepsilon), \text { with } \quad w=\hat{W}(Z, \xi ; \varepsilon), p=\hat{P}(Z, \xi ; \varepsilon),
$$

and we note that

$$
c+V+\beta z=\beta \sqrt{\varepsilon} Z .
$$

The governing equations, (6.1)-(6.3), near to the critical layer in Region I, therefore become

$$
\begin{aligned}
& (-\beta Z+\hat{U}) \hat{U}_{\xi}+\hat{W}\left(-\beta+\hat{U}_{Z}\right)+2 \Omega \hat{W}=-\hat{P}_{\xi}, \\
& \varepsilon\left\{(-\beta Z+\hat{U}) \hat{W}_{\xi}+\hat{W} \hat{W}_{Z}\right\}-2 \Omega \hat{U}=-\hat{P}_{Z}, \\
& \hat{U}_{\xi}+\hat{W}_{Z}=0 .
\end{aligned}
$$

To proceed, we introduce the stream function appropriate to this region (so that $\hat{U}=\hat{\Psi}_{Z}, \hat{W}=-\hat{\Psi}_{\xi}$ ), and then the leading-order problem here, as $\varepsilon \rightarrow 0$, is given by

$$
\left(\hat{\Psi}_{Z}-\beta Z\right) \hat{\Psi}_{Z \xi}-\hat{\Psi}_{\xi}\left(-\beta+\hat{\Psi}_{Z Z}\right)-2 \Omega \hat{\Psi}_{\xi}=-\hat{P}_{\xi} \text { with } \hat{P}_{Z}=2 \Omega \hat{\Psi}_{Z}
$$

Thus $\hat{P}=2 \Omega \hat{\Psi}$ (and any arbitrary functions are absorbed into $\hat{\Psi}$ ), and so we obtain

$$
\left(\hat{\Psi}_{Z}-\beta Z\right)\left(\hat{\Psi}_{Z}-\beta Z\right)_{\xi}-\hat{\Psi}_{\xi}\left(\hat{\Psi}_{Z}-\beta Z\right)_{Z}=0
$$

or

$$
\left(\hat{\Psi}_{Z}-\beta Z\right)\left(\hat{\Psi}_{Z Z}\right)_{\xi}-\hat{\Psi}_{\xi}\left(\hat{\Psi}_{Z Z}\right)_{Z}=0
$$

when expressed in terms of the vorticity, $\hat{\Psi}_{Z Z}$. Thus $\hat{\Psi}_{Z Z}=F\left(\hat{\Psi}-\frac{1}{2} \beta Z^{2}\right)$, and the appropriate solution of this must match to the solution valid outside (away from) the critical layer (see (6.4)), 
namely

$$
-\frac{1}{2} \beta Z^{2}+\left(A \mathrm{e}^{k z_{c}}+B \mathrm{e}^{-k z_{c}}\right) \frac{1}{k} \cos (k \xi),
$$

i.e. $\hat{\Psi}$ must match to $\left(A \mathrm{e}^{k z_{c}}+B \mathrm{e}^{-k z_{c}}\right) \frac{1}{k} \cos (k \xi)$ as $Z \rightarrow \infty$. The stream function in the moving frame in the critical layer, to leading order, is therefore

$$
\hat{\Psi}=-\frac{1}{2} \beta Z^{2}+\left(A \mathrm{e}^{k z_{c}}+B \mathrm{e}^{-k z_{c}}\right) \frac{1}{k} \cos (k \xi),
$$

and the streamlines are then expressed as

$$
\left(A \mathrm{e}^{k z_{c}}+B \mathrm{e}^{-k z_{c}}\right) \frac{1}{k} \cos (k \xi)-\frac{1}{2} \beta Z^{2}=\text { constant },
$$

which recovers the classical Kelvin cats'-eyes pattern.

This analysis has demonstrated how the systematic formulation of the problem - albeit a problem with underlying oceanographic modelling-assumptions - using conventional and familiar techniques of classical fluid mechanics, enables the critical layers to be identified and described.

\subsection{Long (and moderately long), weakly nonlinear waves}

In our discussion of the linear problem, we emphasised the fact that the general results hold for arbitrary wave number, but that we could make headway by approximating our exact dispersion relation for suitably chosen wave lengths. In particular, long, or moderately long ('intermediate'), waves were of the greatest interest. If we start with the requirement that we should limit the discussion to long waves, then obviously there will be some immediate simplifications but, more importantly, this opens the door to an investigation of the possible effects of nonlinearity using techniques that are familiar in fluid mechanics. Further, as we shall outline, this also enables us to interpret the various (long) waves in a slightly different way, illuminating some aspects of the problem.

The starting point here is to regard the amplitude parameter, $\varepsilon$, as the crucial measure which we shall use to compare the sizes of the various properties of the flow. We consider waves that propagate at speed $c$, and incorporate the wave length, in terms of $\varepsilon$, by defining an appropriate travelling-wave coordinate; in addition, we will be interested in how the waves evolve on some suitable timescale, so we introduce

$$
\xi=\varepsilon^{m}(x-c t), \quad \tau=\varepsilon^{m+n} t, \quad w \rightarrow \varepsilon^{m} w .
$$

For long waves we require $m>0$, and we expect $n>0$ (but this is not imposed, at this stage); the transformation on $w$ is to ensure consistency with the equation of mass conservation (and we have opted not to introduce a new symbol). Our equations, (3.12)-(3.19), become

$$
\begin{aligned}
& \varepsilon^{n} u_{\tau}+[U(z)-c+\varepsilon u] u_{\xi}+w\left[U^{\prime}(z)+\varepsilon u_{z}\right]+2 \Omega w=-p_{\xi}, \\
& \varepsilon^{2 m}\left\{\varepsilon^{n} w_{\tau}+[U(z)-c+\varepsilon u] w_{\xi}+\varepsilon w w_{z}\right\}-2 \Omega u=-p_{z}, \\
& u_{\xi}+w_{z}=0
\end{aligned}
$$

with

$$
\begin{aligned}
& P(z)+\varepsilon p=P_{a} \quad \text { on } \quad z=\varepsilon \eta, \\
& w=\varepsilon^{n} \eta_{\tau}+[-V-c+\varepsilon u] \eta_{\xi} \text { on } \quad z=\varepsilon \eta
\end{aligned}
$$


and

$$
\begin{aligned}
& w=\varepsilon^{n} H_{\tau}+[W-c+\varepsilon u] H_{\xi} \quad \text { on } \quad z=-1+\varepsilon H, \\
& p_{a}-p_{b}=-r(1-2 \Omega W) H \quad \text { on } \quad z=-1+\varepsilon H,
\end{aligned}
$$

with

$$
w=0 \quad \text { on } \quad z=-d(\varepsilon) .
$$

The position of the bed of the ocean, $z=-d(\varepsilon)$, has been re-expressed to accommodate the parameter choice that we are at liberty to make in this re-interpretation of the problem; we will return to the choices for $d$ later. The condition of irrotationality of the perturbation, (4.2), becomes

$$
u_{z}-\varepsilon^{2 m} w_{\xi}=0 .
$$

Of course, we need to identify some balance of terms in the governing equations in order to proceed. The timescale on which the nonlinearity will contribute to the evolution of the wave is $\varepsilon^{-1}$, relative to the size of the travelling-wave coordinate; this is clear on physical grounds, and self-evident from the governing equations: we choose $n=1$. However, the choice of $m$ is less straightforward, and hinges on the particular problem under consideration. If $m>1 / 2$, then on the timescale just fixed, the nonlinearity will dominate; if $m=1 / 2$, the effects of nonlinearity and dispersion are the same size and a balance can occur, along the lines of KdV theory; finally, if $m<$ $1 / 2$, dispersion will dominate on this timescale. These are quite familiar considerations. Probably the case of most mathematical interest - but not necessarily of most practical relevance - is the $\mathrm{KdV}$ scenario, but this is far from a routine calculation of this type, because of the complexity of the flow field. And this is not the end of the complications in this problem: the size of $d$ will play a significant rôle, because it can be used to define long and moderately long waves. We leave the complete development of a $\mathrm{KdV}$ theory to one side; it is out-of-place to produce all the intricate details in this article which is aiming to highlight the way in which these problems can be tackled using conventional methods. (Of course, the character of the $\mathrm{KdV}$ problem, in this complicated flow structure, could well uncover some new and interesting phenomena, but that is another story; for some background on KdV theory, see [11].) We will, however, begin the process that is required, no matter what particular long-wave solutions are being considered.

The leading-order problem, for any of the choices with $m>0$, mirrors the linear calculation that we described earlier, but with some important differences, that we shall briefly describe here. The leading-order problem is easily constructed (but note that the move to higher order, as would be needed for the corresponding $\mathrm{KdV}$ calculation, requires careful treatment of the boundary conditions: Taylor expansions will have to be constructed). From equations (6.6)-(6.13), we obtain the equations and boundary conditions that define the leading order as $\varepsilon \rightarrow 0$ :

$$
\begin{aligned}
& {[U(z)-c] u_{\xi}+w U^{\prime}(z)+2 \Omega w=-p_{\xi},} \\
& 2 \Omega u=p_{z}, \\
& u_{\xi}+w_{z}=0,
\end{aligned}
$$

with

$$
\begin{array}{lll}
p=(1+2 \Omega V) \eta & \text { on } & z=0 \\
w=-(V+c) \eta_{\xi} & \text { on } & z=0
\end{array}
$$


also

$$
\begin{aligned}
& w=(W-c) H_{\xi} \quad \text { on } \quad z=-1, \\
& p_{a}-p_{b}=-r(1-2 \Omega W) H \quad \text { on } \quad z=-1,
\end{aligned}
$$

and

$$
w=0 \quad \text { on } \quad z=-d(\varepsilon) .
$$

In this set, (6.14)-(6.21), we have used the properties that pertain to our chosen profile, (4.1), but one of the strengths of this approach (which, of course, is only valid for long waves in the sense of $\varepsilon \rightarrow 0$ in (6.5)) is that the calculation can be carried through for any $U(z)$. The resulting formulation is then expressed in terms of integrals of the type that lead to the Burns condition; see (5.4) and the results that we quote below. As we have noted before, $p$ must be replaced by $p /(1+r)$ in $(6.14)$ and (6.15) when the region below the thermocline is considered.

The solution to the problem above the thermocline, at leading order as $\varepsilon \rightarrow 0$, is:

$$
\begin{aligned}
& w=(U-c)\{1-(1-2 \Omega c) I\} \eta_{\xi} ; \\
& u=\{(1-2 \Omega c)(U-c) I-U\}^{\prime} \eta ; \\
& p=((1-2 \Omega c)\{1+2 \Omega(U-c) I\}-2 \Omega c(U-c)) \eta \\
& H=\left\{1-(1-2 \Omega c) I_{-1}\right\} \eta
\end{aligned}
$$

where

$$
I(z)=\int_{z}^{0} \frac{\mathrm{d} z^{\prime}}{\left(U\left(z^{\prime}\right)-c\right)^{2}} \quad \text { for } \quad 0 \geq z \geq-1, \quad \text { and } \quad I_{-1}=\int_{-1}^{0} \frac{\mathrm{d} z^{\prime}}{\left(U\left(z^{\prime}\right)-c\right)^{2}} .
$$

Below the thermocline, the corresponding solution (written with the circumflex) is

$$
\begin{aligned}
& \hat{w}=(U-c)\left\{H_{\xi}-A_{\xi} J\right\} ; \\
& \hat{u}=A\{(U-c) J\}^{\prime}-U^{\prime} H ; \\
& \frac{\hat{p}}{1+r}=\{1+2 \Omega(U-c) J\} A-2 \Omega(U-c) H ;
\end{aligned}
$$

where

$$
J(z)=\int_{z}^{-1} \frac{\mathrm{d} z^{\prime}}{\left(U\left(z^{\prime}\right)-c\right)^{2}} \quad \text { for } \quad-1 \geq z
$$

and $A(\xi, \tau)$ is an unknown function; the pressure-difference condition across the thermocline gives

$$
(1+r) A=\left\{1-2 \Omega W+2 \Omega(1-2 \Omega c)(W-c) I_{-1}\right\} \eta+\{2 \Omega(W-c)+r(1-2 \Omega c)\} H .
$$

Throughout these expressions, $U(z)$ is that prescribed in (4.1) (because we have introduced the specific parameters $V$ and $W$, but the form for general $U(z)$ is evident). At this stage, we have one boundary condition to impose (which, in principle, will determine $A$ ): (6.21), the condition on the bed of the ocean.

We may apply this boundary condition directly on the expression for $\hat{w}$, and thereby find $A$ and then also the complete solution (to leading order) as described by (6.26)-(6.28). However, this is 
possible only if $d=\mathrm{O}(1)$ as $\varepsilon \rightarrow 0$ i.e. the waves are much longer than the total depth, because the wavelength is $\mathrm{O}\left(\varepsilon^{-1 / 2}\right)$ : this is the case of long waves. We then find that

$$
A=\left(\frac{c^{2}(c-W)^{3}}{c^{2}\{(n-1)(c-W)-m W\}+(d-n)(c-W)^{2}}\right) H .
$$

When this expression is used in (6.29), and $H$ and $\eta$ eliminated between the resulting equation and (6.25), we recover the dispersion relation that was used earlier for long waves (and we also get the correct coupling between the two sets of waves). However, if $d^{-1}=\mathrm{o}(1)$ as $\varepsilon \rightarrow 0$, then the above evaluation is inadmissible, although we observe that this amounts to $A \rightarrow 0$ which happens to produce the correct dispersion relation! This suggests that the matching to a new asymptotic region where $z=\mathrm{O}(d(\varepsilon))$ is possible only if $A=0$ - and this turns out to be the case. Nevertheless, in order to complete the construction of the solution for $d$ s that increase as $\varepsilon \rightarrow 0$, we must introduce a scaled region below $z=\mathrm{O}(1)$ which will take us down to the bed of the ocean; matching is then necessary to determine $A(\xi)$. This will provide the solution for our intermediate long-wave case if we choose $d \varepsilon^{1 / 2} \rightarrow \infty$ as $\varepsilon \rightarrow 0$, but we see that we can also extract the details for the wavelengths consistent with $\varepsilon \rightarrow 0$ and $d \rightarrow \infty$, such that $d \varepsilon^{1 / 2} \rightarrow 0$; these waves are intermediate between long and intermediate long!

It is evident from the above analysis that there is a lot of accessible structure, but this still amounts to a reconsideration of the linear problem (albeit from a rather different standpoint). However, in all that has been presented above, although $\eta$ and $H$ are proportional, we know nothing more about these wave solutions. To obtain this information, we must work to the next order, and this will include higher-order matching to the region which extends down to the bed (if $d$ is larger than $\mathrm{O}(1)$ ). If we select $m=1 / 2$, this is equivalent to performing the classical derivation of the $\mathrm{KdV}$ equation, but in a far more complex scenario (as we have demonstrated). The method and principles underlying this calculation are clear, but there are many delicate issues that have to be addressed on the way. It is also evident that there are many different cases, some of which may provide intriguing mathematical challenges, and some may be of practical significance. All this is left as an exercise for the future.

\section{Conclusions}

We have shown how a theory, based on simple fluid mechanical principles, can be developed for the quite complicated flows that are typical of the Pacific equatorial regions. Although we have used inviscid theory, the inclusion of a background vorticity - albeit containing regions of constant vorticity - has enabled us to model the main characteristics of the EUC that are observed in the Pacific Ocean. This accommodates the wind-driven surface layer (moving westwards) and the EUC core that moves to the East, and a little below the surface. Further, we have embedded a thermocline within this core. The non-dimensionalisation and scaling then produce a consistent linearisation as a (wave) perturbation on this background state. Without the need to make any additional assumptions, we have reported the development of a complete description of the linear wave problem, for arbitrary wavelengths and with the inclusion of a number of parameters that represent the physical system and the details of the background flow field. Although the details (which we did not present here), perhaps not surprisingly, are algebraically somewhat involved, the calculations are altogether routine. We have outlined how it is possible to extract simple, useful information, by approximating for various wavelengths. The agreement with the measured speeds of these various waves is quite 
encouraging. Furthermore, we have demonstrated that there is significant attenuation at the surface, in the case of long waves, of any waves on the thermocline. But all this is really no more than introduction to what the full set of scaled equations can tell us.

One of the great successes, and strengths, of a careful treatment of a problem in fluid mechanics is the wealth of detail embodied in the governing equations - even the inviscid version due to Euler. To exemplify this, we have shown how the appearance of critical levels (predicted by the linear theory) can be examined, resulting in a detailed description of the flows in these critical layers. This is regarded as a standard development nowadays, but it is encouraging to see it play a rôle in a very complex flow, and one for which observations confirm the existence of critical layers in the neighbourhood of the Pacific EUC. Whereas the description of critical layers uses nonlinearity in a local sense, the evolution of waves over long distances and large times requires the use of the nonlinearity on a grander scale. Although we have not given a complete presentation of this aspect - this article is certainly not the place for such an extensive discussion - we have indicated how the ideas will unfold. One result of the formulation of the problem, specifically for suitably long waves, is the prospect of obtaining information more readily than in the general linear case, and also for any chosen background flow. This problem has a nice asymptotic structure in the case of a depth that increases as the parameter $\varepsilon$ decreases, and this has to be embedded within an analysis that is extended beyond the first term, in order to extract the nonlinear and dispersive properties of the wave.

In conclusion, this exercise has demonstrated how effective classical fluid mechanics can be: it has produced a detailed and comprehensive analysis of a flow which contains many physical elements. The formulation has also allowed us to introduce a careful development of two aspects of the nonlinearity inherent in the system.

\section{Acknowledgments}

The author is very grateful for the on-going encouragement of Prof. Adrian Constantin, and for financial support from the ERC Advanced Grant 'Nonlinear studies of water flows with vorticity'.

\section{References}

[1] D. J. Benney and R. F. Bergeron, A new class of nonlinear waves in parallel flows, Stud. Appl. Math. 48 (1969), 181-204.

[2] C. Bosc and T. Delcroix, Observed equatorial Rossby waves and ENSO-related warm water volume changes in the equatorial Pacific Ocean, J. Geophys. Res.-Oceans 113 (2008), C06003.

[3] T.-L. Chiang and T. Qu, Subthermocline eddies in the Western Equatorial Pacific as shown by an eddyresolving OGCM, J. Phys. Oceanogr. 43 (2013), 1241-1253.

[4] A. Constantin, Nonlinear Water Waves with Applications to Wave-Current Interactions and Tsunamis (SIAM, Philadelphia, 2011).

[5] A. Constantin, An exact solution for equatorially trapped waves, J. Geophys. Res.-Oceans 117 (2012), C05029.

[6] A. Constantin, Some nonlinear, equatorially trapped, nonhydrostatic internal geophysical waves, $J$. Phys. Oceanogr. 44 (2014), 781-789.

[7] A. Constantin and R. S. Johnson, The dynamics of waves interacting with the Equatorial Undercurrent, Geophys. Astrophys. Fluid Dynam. 109 (2015), 311-358.

[8] A. V. Fedorov and J. N. Brown, Equatorial waves, in Encyclopedia of Ocean Sciences, Academic Press (Ed. J. Steele), 2009, 3679-3695.

[9] T. Gerkema, J. T. F. Zimmerman, L. R. M. Maas and H. van Haren, Geophysical and astrophysical fluid dynamics beyond the traditional approximation, Reviews of Geophysics 46 (2004), 1-33. 
[10] D. Henry, An exact solution for equatorial geophysical water waves with an underlying current, Europ. J. Mech. B/Fluids 38 (2013), 18-21.

[11] R. S. Johnson, A Modern Introduction to the Mathematical Theory of Water Waves (Cambridge University Press, Cambridge, 1997).

[12] G. C. Johnson and M. J. McPhaden, Equatorial Pacific ocean horizontal velocity, divergence, and upwelling, J. Phys. Oceanogr. 31 (2001), 839-849.

[13] W. S. Kessler, Intraseasonal variability in the oceans, in Intraseasonal variability of the atmosphereocean system (Eds. W. K. M. Lau and D. E. Wallser), Springer, New York, 2005, 175-222.

[14] W. S. Kessler and M. J. McPhaden, Oceanic equatorial waves and the 1991-1993 El Ninõ, J. Climate 8 (1995), 1757-1774.

[15] P. H. LeBlond and L. A. Mysak, Waves in the Ocean (Elsevier, Amsterdam, 1978).

[16] S. A. Maslowe, Critical layers in shear flows, Ann. Rev. Fluid Mech. 18 (1986), 405-432.

[17] J. P. McCreary, Modeling equatorial ocean circulation, Annu. Rev. Fluid Mech. 17 (1985), 359-409.

[18] M. J. McPhaden and J. A. Proehl and L. M. Rothstein, The interaction of equatorial Kelvin waves with realistically sheared zonal currents, J. Phys. Oceanogr. 17 (1986), 1499-1515.

[19] W. D. Smyth and J. N. Moum and J. D. Nash, Narrowband oscillations in the upper equatorial ocean. Part II: Properties of shear instabilities, J. Phys. Oceanogr. 41 (2011), 412-428.

[20] W. T. M. Verkley, On the beta plane approximation, J. Atmos. Sci. 47, 2453-2460. 